she appeared to be too ill to sit up. The wound does not look so healthy; several sloughs have separated. There is no sign of abdominal tenderness. After this the patient became gradually weaker, could with difficulty be persuaded to take food, and the laboured breathing increased. She died four days later.

Necropsy.-There was a large patch of croupous pneumonia at the left base, and general broncho-pneumonia, with some pleurisy. The right lung was nornal. In the abdomen there was no sign of peritonitis. A piece of ileum about twelve inches from the ileo-crcal valve was firmly adherent to the abdominal wound. The femoral ring on the right side, through which the piece of strangulated intestine had descended, was completely closed, and there was no piece of intestine or omentum adherent to it.

Remarks by Mr. BRUCE ClARKE.--This case affords a good illustration of the value of flushing out the peritoneum, and of the completeness with which it can be effected when so large an amount of fiecal matter has been poured into it, as well as of the possibility of dealing successfully with so serious an accident as rupture of the intestine during the course of an operation for strangulated hernia. Had not an attack of pneumonia supervened, there seems every probability that complete recovery would have taken place.

\section{EXE AND EAR HOSPITAL, BALTIMORE, U.S.A.} THE TREATMENT AFTER CATARACT OPERATIONS.

(Under the care of Dr. JULIAN J. CHISOLM.)

THIs account of a method of treatment after the operation for cataract cannot fail to be of interest to our readers, differing as it does from that employed in this country.

This hospital was opened for the treatment of patients twelve years since. During this period 1050 cataract operations have been performed. Up to 1886 there was nothing special in the treatment differing from that pursued elsewhere. The patients were treated in dark rooms, with both eyes heavily bandaged, and were confined to bed with the usual bodily restraints in such general use. Four vears ago modifications in the after-treatment were introduced, until now the course so successfully carried out is altogether different from that pursued in most special hospitals. The first absolu te change is that the eyes are no longer bandaged, and only one eye is closed. Notwithstanding the many arguments on the subject, it is found by experience, in upwards of 400 cataract extractions, that the use of the ancovered eye does not interfere with the thorough and rapid healing of the cut cornea of the eye operated upon. The patient is, therefore, allowed to enjoy the great comfort of using this eye as he did prior to the operation. If the movements of this eye, when put to its ordinary duties, did not disturb the healing process in the cornea of the other, then the use of the arms and leos should not do so. Experience has shown this to be a natural and safe conclusion. It has done away with all bodily restraints and bed treatment. Simultaneously with the liberty allowed to patients was the admission of more light into the sick chamber. Instead of closed shutters, an ordinary window shade, to exclude sunlight, is found by far the better for the patient, to say nothing about the comfort to the attendants. All of these radical changes in the aftertreatment of cataract cases had been brought about from the adoption of a simple dressing for the eye operated upon. It consists of a piece of soft silk isinglitss adhesive plaster an inch and a half long and one inch wide. After the operation for cataract extraction is completed, now without iridectomy, a $\frac{1}{2}$ per cent. solution of eserine is instilled, and both eyes are closed. The adhesive strip is put over the closed lids of the eye operated upon, its length extending from just under the brow to the cheek. It is made damp by a few drops of water, and in its limp condition, aided by a few strokes of a shell spoon, it adapts itself perfectly to the irregularities of the lid surface, fixing every lash down upon the cheek. Being an inch wide, it leaves exposed the angles of the lids, permitting the escape of secretions, and also allowing some drops of eserine to be instilled at bedtime. The plaster is diaphanous, so that the condition of the lids can be determined at the daily visits, without disturbing the adhesive dressing. When the adbesive strip dries, which it does in a few minutes, by its firm hold it has converted the two lids into one continuous septum, a natural mould over the wounded eyeball, supporting and sustaining the perfect adjustment of the lips of the corneal wound as no other dressing can. The other eye can now be opened for use without fear of disturbing the one that is shut in. An every-day familiarity has established so much confidence in the efficacy of this dressing that the patient is literally turned loose to follow out pretty much his own inclinations. He walks unaided from the operating-room and his chamber. He lies on the bed or sits up according to his own wishes. He goes to bed when the proper time comes, making his own toilet, unaided by the attendants. He partakes of the regular solid meals of the hospital diet table. He walks and talks at will, using the uncovered eye as he did prior to the operation. By the fifth day, and sometimes on the fourth, the strip of plaster applied at the time of operation is removed, and after that the eye is left uncovered. A few drops of a 1 per cent. solution of atropia are daily instilled to break up any adhesions which may have formed between the iris and the capsule of the lens. Smoked glasses and brow shades are not now used in the hospital since the abandoning of dark rooms. Most frequently, when patients are discharged from the hospital on the fourteenth day after operation, they go out into the street without protection, smoked glasses being seldom required. The experience of the past four years in this hospital has conclusively shown that most of the sensitiveness of eyes operated upon for cataract is caused by the exclusion of light and the treatment in dark rooms. Healthy eyes submitted to this confining treatment will show the same weeping when the bandages are removed as those operated upon. Since abandoning dark rooms and confining compresses red weeping eyes are rarely seen in the hospital wards. The great success of this non-restraining treatment is well shown in the fact that last year out of 116 cataract extractions, made as patients applied for operation in every varied condition of general health, only two eyes were lost. Such good results were not secured at the hospital in former years when the most careful restraining treatment was carefully carried out

\section{altedurad Sorreties.}

\section{EPIDEMIOLOGICAL SOCIETY.}

\section{Enteric Fever in Indic.}

AT an ordinary meeting held on May 14th, Sir Thomas Crawford, K.C.B., M.D., President, in the chair,

Surgeon-Major R. PRINGLE, M.D., read a paper on Enteric Fever in India, treating successively of (1) its increase, (2) the causes of this increase, (3) the remedies, and (4) the probable consequences. The first question to be deciled was, he said, whether this increase were real or apparent-i.e., due to the incorrect diagnosis and nomenclature of cases of malarial fever, occurring for the most part among young soldiers recently arrived, imprudent or intemperate, and remittentfevers assuming a continued type. This last was, indeed, the conclusion of the Sanitary Commissioners, but it reflected gravely on the knowledge and ability of medical officers of great experience at large military stations, and could not be allowed to pass unchallenged, especially since the lay press, adopting the views of the Conmissioners, had expressed itself very strongly on the supposed incompetence, negligence, or worse, of the medical service generally. Thus the Pioneer, in an article entitled "The Confusion of Fevers," commenting on the fact that in the garrisons at Meerut and Lucknow, of equal strength, there were last year fifty and fifty-one cases of fever respectively, with five deaths at each place, but that while the whole at the former, only thirteen at the latter were returned as enteric, assuming that the conditions were identical, charged the medical officers with "inventing enteric cases out of their own imaginations," whereas this proportion of malarial and enteric cases was precisely what anyone acquainted with the very different sanitary conditions of the two places would expect. The increase of enteric fever was, he maintained, real and indisputable, and its cause to be found in the almost universally polluted water supply. Young men, indeed, were there, as here, more susceptible to enteric fever; but to assign youth as the cause was as irrational as it would be to do so were a number of unvaccinated infants, exposed to small-pox, to contract that disease. The water-supply 